\title{
A Substantial Response from Adding Palbociclib to Endocrine Therapy in Brain Metastasis from Hormone Receptor-Positive, HER2-Negative Breast Cancer: Case Reports
}

\author{
Atlal Abusanad ${ }^{a}$ Hashem Al Hashem $^{b}$ \\ aDivision of Medical Oncology, Internal Medicine Department, Faculty of Medicine, \\ King Abdulaziz University, Jeddah, Saudi Arabia; ${ }^{b}$ Medical Oncology, Department of \\ Hemato-Oncology, King Saud Medical City, Riyadh, Saudi Arabia
}

\section{Keywords}

Breast cancer - Brain metastasis - Palbociclib - Endocrine therapy · Hormone-sensitive breast cancer

\section{Abstract}

Brain metastasis (BM) from breast cancer has poor prognosis despite new advances and multi-modality treatments. No current data is guiding the use of palbociclib in the management of hormone receptor (HR)-positive breast cancer patients with BM as these patients were excluded systematically from all phase 3 trials. Here, we report an evident clinical response from combining palbociclib with endocrine therapy in HR-positive, human epidermal growth factor receptor 2 (HER2)-negative breast cancer with BM.

\section{Introduction}

Hormone receptor (HR) positive, human epidermal growth factor receptor 2 (HER2) negative is the most common subtype of breast cancer. Although brain metastasis (BM) accounts for $14 \%$ of metastatic disease in HR-positive HER2-negative breast cancer, patients with BM are excluded systematically from clinical trials [1, 2]. Among the widely reported barriers to effective treatment for BM is the limited delivery of therapy across the blood-brain barrier (BBB). Therefore, effective treatment for BM represents a serious unmet medical need. 
Table 1. Timeline of disease progression and interventions in patient 1

\begin{tabular}{|c|c|c|c|c|c|}
\hline Time line & Extracranial metastases & Brain metastasis & Systemic therapy & Radiotherapy & Other intervention \\
\hline Fall 2016 & $\begin{array}{l}\text { Symptomatic bone } \\
\text { metastases and impending } \\
\text { cord compression }\end{array}$ & None & $\begin{array}{l}3 \text { weekly docetaxel (8 cycles) } \\
\text { AI + LHRH } \backslash \text { month } \\
\text { Zolanderic acid } \backslash \text { month }\end{array}$ & $\begin{array}{l}\text { Palliative radiation to } \\
\text { thoracolumbar vertebrae }\end{array}$ & \\
\hline Fall 2017 & Stable bone, no visceral & Symptomatic BM (hydrocephalus) & $\begin{array}{l}\mathrm{AI}+\mathrm{LHRH} \backslash \text { month }+ \\
\text { zolanderic acid } \backslash \text { month }\end{array}$ & SRS & VP shunt \\
\hline Summer 2018 & Stable bone, no visceral & Symptomatic first progression & $\begin{array}{l}\text { AI + LHRH } \backslash \text { month + zolanderic } \\
\text { acid } \backslash 3 \text { months }\end{array}$ & WBRT & $\begin{array}{l}\text { Oral } \\
\text { dexamethasone }\end{array}$ \\
\hline Spring 2019 & Stable bone, no visceral & Second progression & Fulvestrant + palbociclib & Not feasible & $\begin{array}{l}\text { Oral } \\
\text { dexamethasone }\end{array}$ \\
\hline Fall 2019 & Stable bone, no visceral & Marked reduction (30\%) in size & Fulvestrant + palbociclib & & No dexamethasone \\
\hline
\end{tabular}

BM, brain metastasis; AI, aromatase inhibitor; LHRH, luteinizing hormone-releasing hormone; SRS, stereotactic radiosurgery; WBRT, whole-brain radiotherapy; VP, ventriculoperitoneal.

Cyclin-dependent kinases 4 and 6 (CDK4/6) are well-established standard of care for advanced HR-positive, HER2-negative breast cancer [3]. Palbociclib is an orally active, selective, and reversible inhibitor of CDK4 and 6. Palbociclib is approved for the treatment of postmenopausal women with HR-positive, HER2-negative disease in combination with endocrine therapy (ET). It is approved in combination with aromatase inhibitors as an initial treatment based on the results of the PALOMA-1 and PALOMA-2 studies [4, 5]. Palbociclib is also approved with fulvestrant in women with disease progression following first-line ET based on PALOMA-3 [6]. However, patients with known uncontrolled or symptomatic central nervous system metastases were uniformly excluded from these trials. We report here a significant clinical and radiological response to palbociclib in combination with ET in BM from HR-positive, HER2-negative breast cancer in 2 cases.

\section{Case Report 1}

Our patient is a 46-year-old premenopausal female, who was diagnosed with HR-positive, HER2-negative breast cancer with extensive bone metastases in fall 2016. She presented with a left breast mass. An ultrasound-guided biopsy from the mass showed invasive ductal carcinoma, grade III, estrogen receptor positive (95\%), progesterone receptor positive (30\%), and HER2-negative by immunohistochemistry. Metastatic workup consisted of computed tomography (CT) scans of the chest-abdomen-pelvis, and nuclear bone scan showed a metastatic left internal mammary lymph node (LN) and a metastatic left axillary LN, as well as multiple bony deposits in the thoracic and lumbar vertebrae, left iliac bone, and left femur. She had no visceral metastases. She received palliative radiotherapy to the vertebral metastases to preventimpending spinal cord compression. Systemic chemotherapy, consisting of 8 cycles of docetaxel with monthly zoledronic acid injection, was started with excellent subjective and objective responses. She was put on letrozole tablets plus goserelin acetate subcutaneous injections after completion of chemotherapy (Table 1).

In November 2017, she presented with new onset of gait instability, headache, nausea, bilateral ptosis, and blurred vision. Magnetic resonance imaging (MRI) of the brain showed a metastatic brain stem lesion along with hydrocephalus. Hydrocephalus was relieved by the insertion of a ventriculoperitoneal shunt followed by stereotactic radiotherapy to the BM (12 Gy in single fraction). Repeated body CT scans and a nuclear bone scan showed stability of bone lesions with no visceral metastases. As a result of the absence of extracranial disease progression, the same ET was continued. In July 2018, she manifested BM progression, which 

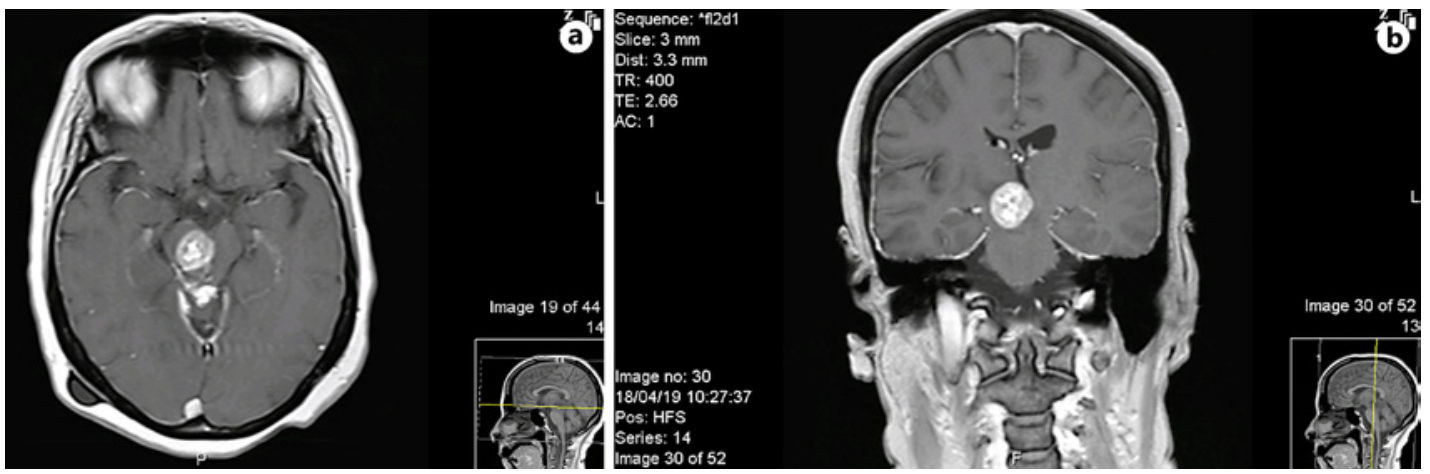

Fig. 1. MRI of the brain in April 2019 of patient 1, which showed a high right mid-brain metastatic lesion. a Axial sections. b Coronal sections.
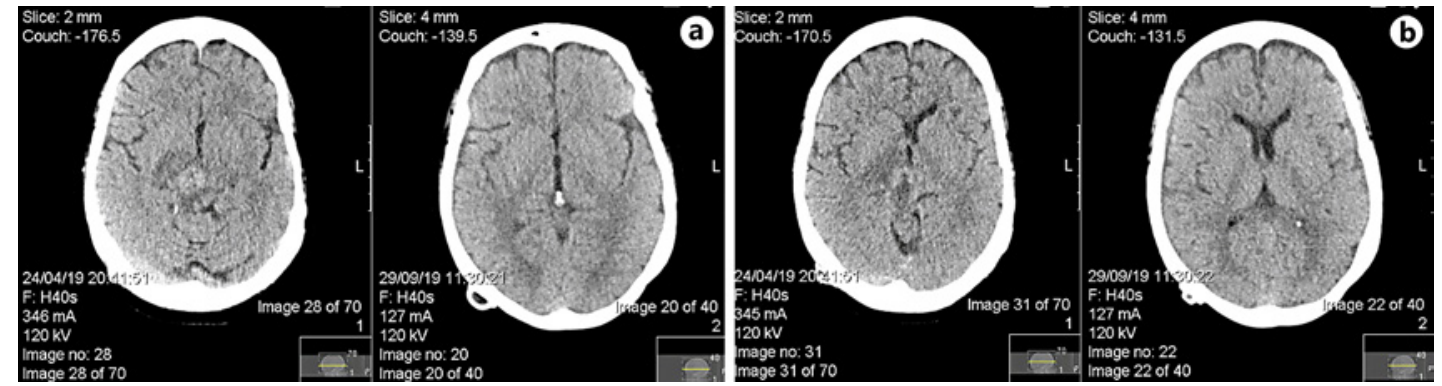

Fig. 2. CT of the brain of patient 1, comparing brain stem lesion in April 2019 (a and $\mathbf{b}$ are different cuts) before palbociclib (right-sided images in $\mathbf{a}$ and $\mathbf{b}$ ) with lesion in September 2019 while on palbociclib (leftsided images in $\mathbf{a}$ and $\mathbf{b}$ ). Noticeable resolution of the lesion and surrounding edema with normalization of the midline structure and the lateral ventricle can be seen.

was confirmed on follow-up brain MRI. Consequently, whole-brain palliative radiotherapy (WBRT) was administrated. Again, repeated body imaging showed stability of extracranial disease on letrozole, and so it was maintained in combination with a luteinizing hormonereleasing hormone agonist.

In April 2019, nearly 9 months later, she developed a second progression in BM manifested with worsening of the previously mentioned neurological symptoms and an increase in the size of the metastatic brain stem lesion on follow-up brain MRI (Fig. 1). Nonetheless, extracranial bone disease remained stable with no evidence of visceral metastases. The patient's performance status declined from 1 to 2 (WHO scale). Neither surgical intervention nor farther irradiation to the brain was feasible, while systemic chemotherapy was deemed unsafe. As a result, palbociclib (125 mg once daily for 21 days) combined with fulvestrant injection (500 mg IM on day 1, 15, and 29, then monthly) were prescribed. She completed 20 weeks on the combination and manifested an excellent subjective response with neurological symptoms improvement. Follow-up CT of the brain showed a substantial reduction in the size of the brain stem lesion with marked improvement of the surrounding brain edema (Fig. 2). She has been followed up with periodic clinical evaluation and laboratory investigations. Treatment with palbociclib plus fulvestrant was tolerated well with no complications. She continues on the treatment mentioned above to the date of the preparation of this report. 


\section{Case Reports in Oncology}
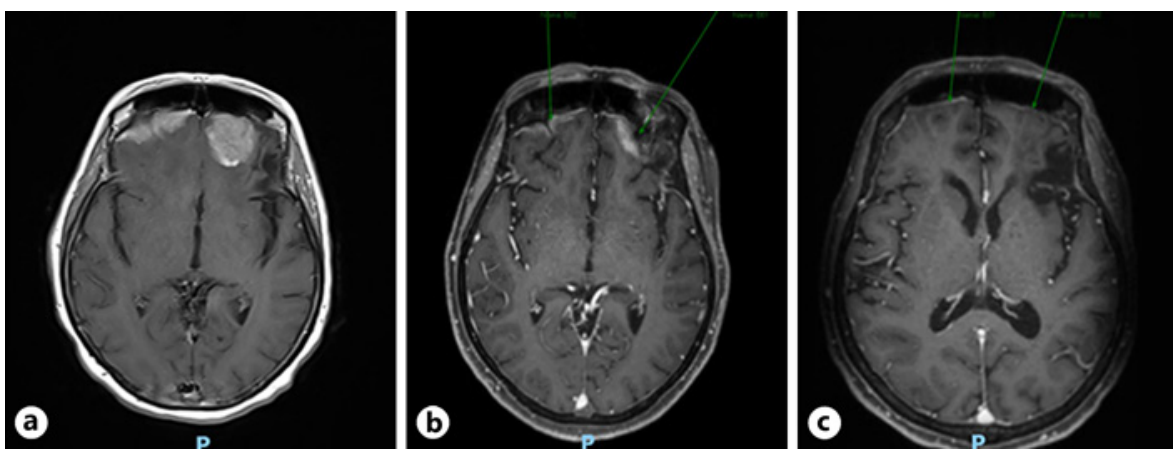

Fig. 3. CT of the brain of patient 2: at the time of diagnosis with BM, October 2019 (a), follow-up CT of the brain while on palbociclib and endocrine therapy shows reduction in BM, April 2020 (b), and continuing the same treatment for 11 months with complete resolution of BM, September 2020 (c).

\section{Case Report 2}

The second patient is a 57-year-old postmenopausal woman diagnosed 12 years ago (in 2008) with locally advanced, HR-positive, HER2-negative right breast cancer. She was treated by modified radical mastectomy and axillary LN dissection followed by 6 cycles of anthracyclines and taxanes chemotherapy, adjuvant radiation therapy, followed by 5 years of tamoxifen, which was completed in 2014.

In October 2019, she presented to the emergency department with a history of right lower limb weakness associated with dizziness, gait instability, and a history of 2 falls; otherwise, there was no history of visual disturbance, seizure, or loss of consciousness. There was no vomiting, and she had no fever. On examination, her Glasgow coma scale was 15/15, the power was $2+/ 5$ in the right lower limb, and $4+/ 5$ in the right upper limb. There was right knee hyperreflexia with negative Babinski sign. The left-side examination was normal. There was no facial asymmetry. The patient's performance status was 4 (WHO scale). Workup consisted of brain MRI and CT scans of the chest-abdomen-pelvis, and nuclear bone scan. Brain MRI showed heterogeneous enhancing infiltrative dural masses in the frontal region bilaterally associated with a significant vasogenic edema; innumerable enhancing intra-axial nodules were scattered all over the brain in the infra- and supratentorial parenchyma. CT of the brain at the time of presentation is shown in Figure 3a. Systemic staging was positive for multiple bilateral pulmonary nodules, multiple sclerotic bony lesions, and an incidental finding of acute pulmonary embolism.

She was started on dexamethasone to relive the vasogenic brain edema and was evaluated by the radiation oncology team, who decided that the palliative brain radiation therapy was not indicated at this point. Considering HR-positive, HER2-negative disease with an almost 12-year disease-free interval, palbociclib (125 mg once daily for 21 days of 28-day cycle) combined with letrozole (2.5 mg orally once daily) was prescribed. She tolerated the treatment well, apart from asymptomatic neutropenia, which led to palbociclib dose reduction to $75 \mathrm{mg}$ once daily for 21 days of 28-day cycle.

Clinically, she is recuperating with physiotherapy and her performance status is improving. Follow-up brain MRI and CT in April 2020 (6 months on the treatment) showed interval regression in the enhancing meninges in the bifrontal region with a residual mild enhancing lesion in the right frontal lobe and complete disappearance and resolution of multiple enhancing lesions in both cerebral hemispheres (Fig. 3b). Systemic CT scan also showed interval regression of the pulmonary nodules and of the skeletal metastasis, reflecting 
regression of the disease process and response to the therapy. A recent CT of the brain from September 2020 showed complete resolution of frontal BM (Fig. 3c) while the patient is continuing on palbociclib and ET.

\section{Discussion}

Breast cancer is the second most common solid cancer that metastasizes to the brain, and symptomatic BM occurs in 10-16\% of patients, whereas they are reported as high as $30 \%$ in autopsy [7]. Breast cancer patients who develop BM have a poor prognosis, and survival in the absence of any treatment rarely exceeds 3 months [8]. Treating BM with systemic therapy is known to be challenging due to several factors, especially inadequate delivery of drugs across the BBB [8]. Nonetheless, other treatment modalities, such as radiotherapy and surgical intervention, may not be feasible in certain situations. Altogether these highlight the paramount need for novel therapeutics to manage BM. Paradoxically, clinical trials have been systematically excluding patients with BM, resulting in a paucity of information on the effectiveness of new targeted therapies, such as CKD4/6 inhibitors, in BM from breast cancer. Palbociclib is the first CKD4/6 inhibitor in class, which has been approved in combination with ET for both first- and later-lines advanced/metastatic HR-positive HER2-negative breast cancer [4-6]. The PALOMA 1, 2, and 3 trials excluded patients with BM. So, the ability of palbociclib to cross the BBB in HR-positive HER2-negative breast cancer was not reported before in HR-positive HER2-negative breast cancer with BM.

Palbociclib primarily led to a reduction in brain stem lesions in these cases. The first patient had bone metastases that have been controlled throughout the disease course on ET, and she never developed visceral spread; emphasizing that the tumor responsiveness to ET persisted. Simultaneously, she had poor response and recurrent progression in BM. This differential response supports the limited delivery of ET across the BBB and, thus, the lack of response even in persistently hormone-responsive tumors. Although our patient received stereotactic radiotherapy and WBRT on different occasions, which may have impaired the permeability of the BBB further, the tumor shrinkage was remarkable. It is important to note that fulvestrant is unlikely to cross the BBB, which is evident by preclinical studies and the lower incidence of hot flushes than the ones associated with tamoxifen $[9,10]$.

The second patient had recurrent brain, visceral (manifested by lung metastasis), and bone metastasis. She had prior sensitivity to hormonal therapy. On the recurrence, she showed excellent response to palbociclib with letrozole in both her brain and extracranial disease. It is noteworthy that abemaciclib has been shown to cross the BBB [11], which supports our observation and may contribute to a class-effect of CDK4/6 inhibitors.

There is significant improvement in treating HR-positive breast cancer with bone and visceral metastases; however, BM treatment is not progressing in parallel. These cases suggest that palbociclib is useful in managing BM from HR-positive, HER2-negative breast carcinoma. It could be considered as a treatment option for patients with HR-positive, HER2-negative breast cancer and BM in the first or subsequent lines of therapy.

\section{Conclusion}

To our knowledge, this is the first report of patients treated with palbociclib plus ET for symptomatic uncontrolled BM from HR-positive, HER2-negative metastatic breast cancer in clinical practice with remarkable response. Clinical trials of novel therapeutics should expand

\section{Karger ${ }^{\prime}=$}


their inclusion criteria to include patients with BM to align with the progressive improvement in the treatment of other metastatic sites.

\section{Acknowledgement}

The authors acknowledge Dr. Osama Elzaffarany for assisting in reviewing the file of the first case.

\section{Statement of Ethics}

Institutional review board approval for case reports and patient chart review was granted. Personal identifiers were concealed as per institutional guidelines. Written informed consent was obtained from the patients for the publication of these case reports and any accompanying images.

\section{Conflict of Interest Statement}

There are no disclosures and no financial and/or nonfinancial competing interests to be declared.

\section{Funding Sources}

No funding was received for this research.

\section{Author Contributions}

Both authors contributed equally to the conceptualization, data collection, writing, and reviewing of the initial and final draft of the manuscript and they approved the final version of the submitted manuscript.

\section{References}

1 Brosnan EM, Anders CK. Understanding patterns of brain metastasis in breast cancer and designing rational therapeutic strategies. Ann Transl Med. 2018;6(9):163.

2 Kotecki N, Lefranc F, Devriendt D, Awada A. Therapy of breast cancer brain metastases: challenges, emerging treatments and perspectives. Ther Adv Med Oncol. 2018;10:1758835918780312.

3 Choi YJ, Anders L. Signaling through cyclin D-dependent kinases. Oncogene. 2014;33(15):1890-903.

4 Finn RS, Martin M, Rugo HS, Jones S, Im SA, Gelmon K, et al. Palbociclib and letrozole in advanced breast cancer. N Engl J Med. 2016;375(20):1925-36.

5 Finn RS, Crown JP, Lang I, Boer K, Bondarenko IM, Kulyk SO, et al. The cyclin-dependent kinase 4/6 inhibitor palbociclib in combination with letrozole versus letrozole alone as first-line treatment of oestrogen receptorpositive, HER2-negative, advanced breast cancer (PALOMA-1/TRIO-18): a randomised phase 2 study. Lancet Oncol. 2015;16(1):25-35.

6 Cristofanilli M, Turner NC, Bondarenko I, Ro J, Im SA, Masuda N, et al. Fulvestrant plus palbociclib versus fulvestrant plus placebo for treatment of hormone-receptor-positive, HER2-negative metastatic breast cancer that progressed on previous endocrine therapy (PALOMA-3): final analysis of the multicentre, double-blind, phase 3 randomised controlled trial. Lancet Oncol. 2016;17(4):425-39.

7 Lin NU, Amiri-Kordestani L, Palmieri D, Liewehr DJ, Steeg PS. CNS metastases in breast cancer: old challenge, new frontiers. Clin Cancer Res. 2013;19(23):6404-18. 
8 Costa R, Carneiro BA, Wainwright DA, Santa-Maria CA, Kumthekar P, Chae YK, et al. Developmental therapeutics for patients with breast cancer and central nervous system metastasis: current landscape and future perspectives. Ann Oncol. 2017;28(1):44-56.

9 Vergote I, JR., Robertson JF. Fulvestrant is an effective and well-tolerated endocrine therapy for postmenopausal women with advanced breast cancer: results from clinical trials. Br J Cancer. 2004;90(Suppl 1):S11-4.

10 Stemmler HJ, Heinemann V. Central nervous system metastases in HER-2-overexpressing metastatic breast cancer: a treatment challenge. Oncologist. 2008;13(7):739-50.

11 Tolaney SM, Sahebjam S, Le Rhun E, Lin NU, Markel Bear M, Yang Z, et al. Abstract P1-19-01: A phase 2 study of abemaciclib in patients with leptomeningeal metastases secondary to HR+, HER2- breast cancer. San Antonio Breast Cancer Symposium; December 4-8, 2018. San Antonio, Texas. 2019;79(4 suppl). 